be explained as follows: Hypertrophy of the anterior pituitary leads $(a)$ to increased formation of creatine and $(b)$ to stimulation of the gonads. The latter factor increases the inhibitory action of the gonads on creatine-creatinine change with the result that some of the excess creatine appears in the urine as such, and some appears in the form of increased creatinine.

On the basis of the above considerations, the following hypothesis is advanced as an explanation of the ereatinuria of pregnancy. Functional hypertrophy of the anterior lobe of the pituitary occurs in pregnancy, and this leads to essentially the same processes as in acromegaly except for the effect of a persistent corpus luteum. It is suggested that the corpus luteum reinforces the inhibitory action of the ovary on the transformation of creatine to creatinine so that all the excess creatine is excreted as such and the creatinine level remains unchanged.

Thus, in the castrated animal the inhibitory action of the gonads is nil, and all excess creatine is excreted as creatinine. In pregnancy the inhibitory action is a maximum, and all excess creatine is eliminated as such. Acromegaly presents an intermediate condition which leads to the appearance of creatine in the urine of males, and an increased excretion of creatinine. In all these conditions the hypertrophy of the anterior lobe of the pituitary, and the production of excess creatine, is a common factor, but the differences in urinary output are due to quantitative differences in the amount and extent of the inhibitory action of the gonads on the transformation of creatine to creatinine in. the muscles.

It is possible that the endocrine factors outlined above, coupled with the probability that the immaturity of the young animal's muscles is associated with a defective capacity to utilise creatine (Powis and Raper $^{3}$ ), may supply a basis for an explanation of the creatinuria during growth.

The hypothesis suggested rests only partly on experimental evidence but it indicates the lines on which future inquiry may profitably be based.

I. SCHRTRE.

H. ZWARENSTEIN.

Department of Physiology,

University of Capetown.

1 Schrire and Zwarenstein, Biochem. J., 26, 118; 1932: 26, $1886 ; 1932$ : in press, 1932 : in press, 1933."

Mirvish and Schrire, Private communication. 1933.
Powis and Raper, Biochem. J., 10,363; 1916.

\section{Experiments on Evaluation of Helium from Radio- active Minerals and Rocks}

IT is very well known that the rate of loss of helium from different radioactive minerals and rocks depends on the dimensions of the surface and on the temperature. When minerals are finely ground, or heated to a high temperature, there is a considerable loss of helium, which can attain about 90 per cent when both of the above mentioned factors are concerned.

Theoretical considerations make it very probable that the amount of helium lost from minerals depends in some cases also on the composition of the gaseous phase which surrounds the mineral or the rock sample. We have proved this assumption experimentally and the results obtained seem of sufficient interest to be recorded.

The amount of helium evolved from different minerals at a given temperature, if this temperature is above a critical one, depends on the presence of hydrogen in the gaseous phase and is the greater the higher the partial pressure of hydrogen. The rate of loss of helium from uraninite (pitchblende) during two hours' heating at $500^{\circ}$ is as follows :in vacuo, 10 per cent; in atmosphere of hydrogen at $25 \mathrm{~mm}$. pressure, 17 per cent; in atmosphere of hydrogen at $100 \mathrm{~mm}$. pressure, 36.5 per cent; in atmosphere of hydrogen at $500 \mathrm{~mm}$. pressure, 60 per cent. The rate of loss from a mineral of the family of euxenite (chlopinite) at $900^{\circ}$ is :-in vacuo, $13 \cdot 3$ per cent ; in atmosphere of hydrogen at $250 \mathrm{~mm}$. pressure, $56 \cdot 1$ per cent.

The influence of hydrogen upon the rate of evolution of helium from minerals is so well marked that small amounts of hydrogen in a gas mixture can be detected by means of this process. A more detailed description of these experiments, and the discussion of the results obtained, will be given in another paper.

State Radium Institute,

V. CHLOPIN.

E. Herling.

E. JoFFÉ.

Leningrad.

Petrogradskaja Storona, Ul. Roentgen 1. Nov. 30 .

\section{Crystal Absorption by Substrates}

In the course of recent experiments it was found, in agreement with French ${ }^{1}$, that suitable polishing destroys the crystalline structure of metallic surfaces. In addition, new and remarkable facts came to light. Thus, we have observed that when certain metal vapours are condensed on a substrate consisting of a polished metallic surface, crystals are formed which, however, rapidly disappear at room temperature. This is borne out by the fact that, whilst the freshly formed deposit gives rise to a characteristic electrondiffraction pattern, the rings more or less rapidly disappear, and that without any appreciable broadening effect. On the other hand, in the case of a crystalline but otherwise similar substrate, the diffraction pattern yielded by the deposit is permanent.

Thus, the stability or otherwise of the deposit crystals is determined by the condition of the substrate. For example, we have found that zinc vapour suitably condensed on a cool, polished copper surface gives rise to an initially brilliant and well-defined electron-diffraction pattern which rapidly fades away, to become extinct within a few seconds. In one such experiment, twelve successive zinc layers were deposited. With each layer except the last the initial crystalline structure vanished at a rate decreasing with each successive deposit. Zine deposited under otherwise similar conditions, but on sputtered or etched copper, or on a previously oxidised and then reduced copper surface, formed a crystalline film the structure of which remained unchanged. It seems to us that these facts afford direct experimental proof of the existence of the Beilby layer.

\section{G. I. Finch.}

A. G. Quarrelt.

J. S. RoEBuck.

Imperial College of Science and Technology.

$$
\text { Dec. } 13 .
$$

${ }^{2}$ Proc. Roy. Soc., A, 140, 637 ; 1933. 